\title{
"Sajino" criados en cautiverio para subsistencia en pueblos indígenas (Loreto, Perú)
}

"Sajino" bred in captivity for subsistence in indigenous peoples (Loreto, Peru)

"Sajino" criado em cativeiro para subsistência de povos indígenas (Loreto, Peru)

- Martha Estela Rengifo Pinedo

martha.rengifo@unapiquitos.edu.pe

Código ORCID: 0000-0001-9147-3302
- Darvin Navarro Torres

darvin.navarro@unapiquitos.edu.pe

Código ORCID: 0000-0002-6808-3151

Universidad Nacional de la Amazonia Peruana- UNAP, Facultad de Ciencias Biológicas, Perú

Artículo recibido 23 de octubre de 2020 / Arbitrado y aceptado 21 de noviembre 2020 / publicado 18 de diciembre 2020

\section{RESUMEN}

El objetivo general en esta investigación es analizar la crianza de sajinos en cautiverio por parte de los pueblos indígenas de Loreto, Perú. La metodología aplicada se basó en investigación cualitativa tomando en consideración la investigación acción. En este particular la investigación se centró en la crianza de los sajinos por parte de cuatro poblaciones indígenas localizados en la región de Loreto. Estas etnias son Kukama-kukamiria, Rio Nanay, Huitoto muruy (Rio Amazonas), Achuar (Rio Pastaza) y Arabela (Rio Arabela), las cuales fueron evaluadas por etapas comprendidas entre el 2002 al 2011. Como conclusión, los pobladores de los pueblos indígenas demostraron conocimientos previos útiles en la crianza del Pecarí tajacu; para la producción de alimentos no se necesitó rozar- talar- quemar el bosque; por el contrario, se colectaron la producción del bosque en pie; basada en el contexto de sostenibilidad e identidad cultural en cada pueblo; de igual manera, se apoyaría esta iniciativa, si habilitamos socialmente el concepto de Unidad Familiar de Subsistencia (UFs), permitiendo adaptarlo al sistema de propiedad comunal en los pueblos indígenas.

Palabras clave: Cría animal; pecarí; población indígena, sostenibilidad

\begin{abstract}
The general objective of this research is to analyze the breeding of sajinos in captivity by the indigenous peoples of Loreto, Peru. The applied methodology was based on qualitative research taking action research into consideration. In this particular, the research focused on the raising of the sajinos by four indigenous populations located in the Loreto region. These ethnic groups are Kukama-Kukamiria, Rio Nanay, Huitoto Muruy (Rio Amazonas), Achuar (Rio Pastaza) and Arabela (Rio Arabela), which were evaluated by stages between 2002 and 2011. In conclusion, the inhabitants of the indigenous peoples they demonstrated useful. previous knowledge in the breeding of the Pecarí tajacu; For the production of food it was not necessary to slash-cut-burn the forest; on the contrary, the production of the standing forest was collected; based on the context of sustainability and cultural identity in each town; Likewise, this initiative would be supported if we socially enable the concept of the Subsistence Family Unit (FUs), allowing it to be adapted to the communal property system in indigenous peoples.
\end{abstract}

Key words: Animal husbandry; peccary; indigenous population, sustainability 


\section{RESUMO}

O objetivo geral desta pesquisa é analisar a criação de sajinos em cativeiro pelos povos indígenas de Loreto, Peru. A metodologia aplicada baseou-se em pesquisa qualitativa considerando a pesquisa-ação. Nesse particular, a pesquisa se concentrou na criação de sajinos por quatro populações indígenas localizadas na região de Loreto. Essas etnias são Kukama-Kukamiria, Rio Nanay, Huitoto Muruy (Rio Amazonas), Achuar (Rio Pastaza) e Arabela (Rio Arabela), que foram avaliadas em etapas de 2002 a 2011. Concluiu-se que os habitantes das cidades indígenas demonstrou conhecimento prévio útil na criação de Pecarí tajacu; Para a produção de alimentos não era necessário cortar - derrubar - queimar a floresta; ao contrário, a produção da floresta em pé foi coletada; com base no contexto de sustentabilidade e identidade cultural de cada cidade; da mesma forma, essa iniciativa seria apoiada, se capacitarmos socialmente o conceito de Unidade Familiar de Subsistência (UF), permitindo sua adaptação ao sistema de propriedade comunal dos povos indígenas.

Palavras-chave: Criação animal; pecari; população indígena, sustentabilidade

\section{INTRODUCCIÓN}

La agricultura familiar es definida mundialmente como un sistema de producción agropecuaria, cuya mano de obra y administración dependen principalmente de los integrantes de la familia. Estas explotaciones de pequeña escala constituyen la forma dominante de agricultura en el mundo y está arraigada a las zonas rurales. Conviene destacar que el concepto de ruralidad en América latina y el Caribe ha venido evolucionado, siendo definido en función de las actividades económicas, sociales e incluso culturales que se desarrollan es un espacio geográfico, se sostiene que en la zona rural se encuentra el 29\% de las personas pobres de América Latina $(1,2)$. Además, se conoce que el $41 \%$ de ellas, están en pobreza extrema y que generalmente es producida por factores multidimensionales (3).

En el Perú, el último censo nacional agropecuario realizado en el 2012 reveló que la superficie agropecuaria es bastante reducida (38,742,465 ha), donde la mayor parte está tipificada como tierras eriazas, bosques $\mathrm{y}$ tierras improductivas de menor valor, ubicados en la región amazónica (4). Asimismo, recogiendo los datos censales del 2012, se afirma que "la agricultura familiar representa el 97\% del total de las más de 2,2 millones de unidades agropecuarias (UA); y en algunos departamentos del país, esta tasa asciende a casi el 100\%" (2).

Del mismo modo, la importancia en términos de extensión de tierras, la cantidad de familias que albergan, su rol en la conservación de la biodiversidad, ambiente, así como sus valores culturales y las organizaciones presentes en estos territorios no forman parte del programa de unidades familiares del estado peruano. Por ello, para el caso Perú se precisa que la agricultura familiar de subsistencia (autoconsumo), genere tecnologías propias en base a sus recursos naturales. En consecuencia, es importante visibilizar a la fauna silvestre y las poblaciones humanas que dependen para su seguridad alimentaria usufructuando el bosque (Pueblos indígenas) en el marco de una ganadería amazónica con especies locales. A nivel de América Latina ya existen iniciativas de crianzas agropecuarias: cría de Hydrochoerus (capibara), Dasyprocta (agutí), Myocastor (coipú), Cuniculus paca (paca), Pecarí tajacu (pecarí o sajino), cuyo consumo se encuentran muy extendidos como carne de monte $\mathrm{u}$ otras denominaciones $(5,6)$. Así mismo, es conocido que la domesticación en el Perú tiene más de 100 años de vida y la diversidad de recursos genéticos es un logro 
de las poblaciones peruanas, se ha domesticado especies de flora $y$ fauna silvestre (7). Así mismo, la extracción de recursos naturales en el país es igualmente antigua.

En la actualidad el Perú, es el único país en Latinoamérica que está exportando pieles de pecarí o sajino como subproducto de la caza de subsistencia (8). Por ejemplo, los ingresos por ventas del cuero de sajino, hasta el año 2017, se observa en la Figura 1. Así mismo, se confirma que los pecaríes, al estar incluidos en los apéndices CITES, son de especial interés del estado y se sostiene que se requieren medidas para su gestión (9). Sin embargo, el estado peruano en la política de expansión agraria promociona la crianza de especies exóticas (ganado vacuno, bubalino, conejos, cabras, ovinos, etc). Los cuales, a la fecha han demostrado su insostenibilidad, como unidades productivas rentables para la amazonia de selva baja, dado que la aptitud del bosque es forestal. No incentivando tecnologías para la cría de pecarí u otros animales obtenidos del bosque (6).

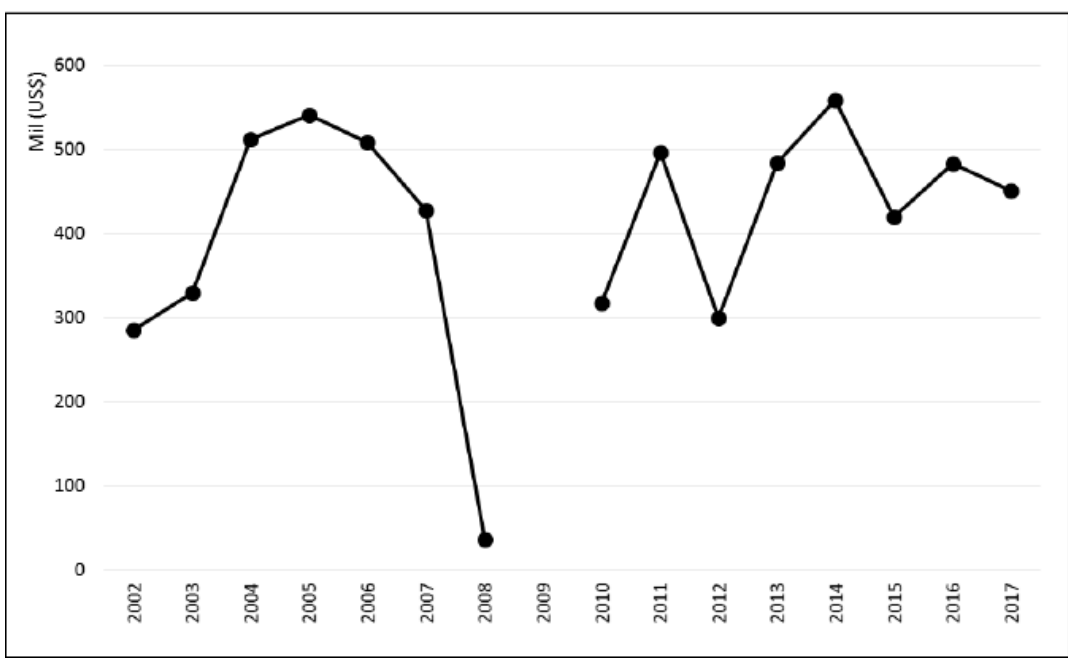

Figura 1. Valor de las exportaciones de cueros de pecaríes (2002 - 2017). Fuente: (10)

En este sentido, el objetivo de la presente investigación es la sistematización de la crianza de "sajino" Pecarí tajacu, Linnaeus 1758 en familias de cuatro pueblos indígenas: Kukama, Huitoto muruy, Achuar, Arabela de la Región Loreto en la Amazonia Peruana. Dicho estudio se realizó entre los años 2002 al 2011 con la finalidad de validar y contribuir a proponer una actividad forestal- agropecuaria familiar de acuerdo con el tipo de bosque, tierra y sobre todo a la identidad cultural en cada pueblo indígena que genere un valor agregado a lo denominado como tierras improductivas. La metodología aplicada se basó una investigación cualitativa tomando en consideración la investigación acción.

\section{MATERIALES Y MÉTODOS}

Dentro de la investigación cualitativa se encuentra el desarrollo de la investigación acción, esta se caracteriza como se detalla a continuación: 
Forma parte de una situación social específica en la cual se ha detectado una problemática; desarrolla un proceso dialéctico en el cual la acción genera conocimiento y la investigación conduce a la transformación; en el proceso trabajan de forma continua y mancomunada los integrantes de la comunidad en estudio, los investigadores, promotores y animadores; la dinámica de participación genera un compromiso, tanto del investigador como del resto de los participantes (11).

En este particular la investigación se centró en un análisis cualitativo de la crianza de los sajinos por parte de los cuatro pueblos Indígenas localizados en la región de Loreto. Siendo Kukama-kukamiria, Huitoto muruy,
Achuar y Arabela los pueblos participantes, los cuales fueron evaluados en etapas entre el 2002 al 2011, donde se incorpora la sostenibilidad. La localización de estas comunidades se muestra en la Figura 2.

Se homogenizó la muestra en tres familias criadoras por comunidad. El análisis de datos se realizó siguiendo lo propuesto por (12) basado en tres momentos Descubrimiento (Taller de sensibilización), Codificación (Capacitación en el manejo de los animales) y Relativización (cría en las unidades familiares de subsistencia). Durante el proceso se utilizó entrevistas semiestructuradas, encuestas y observación de los participantes.

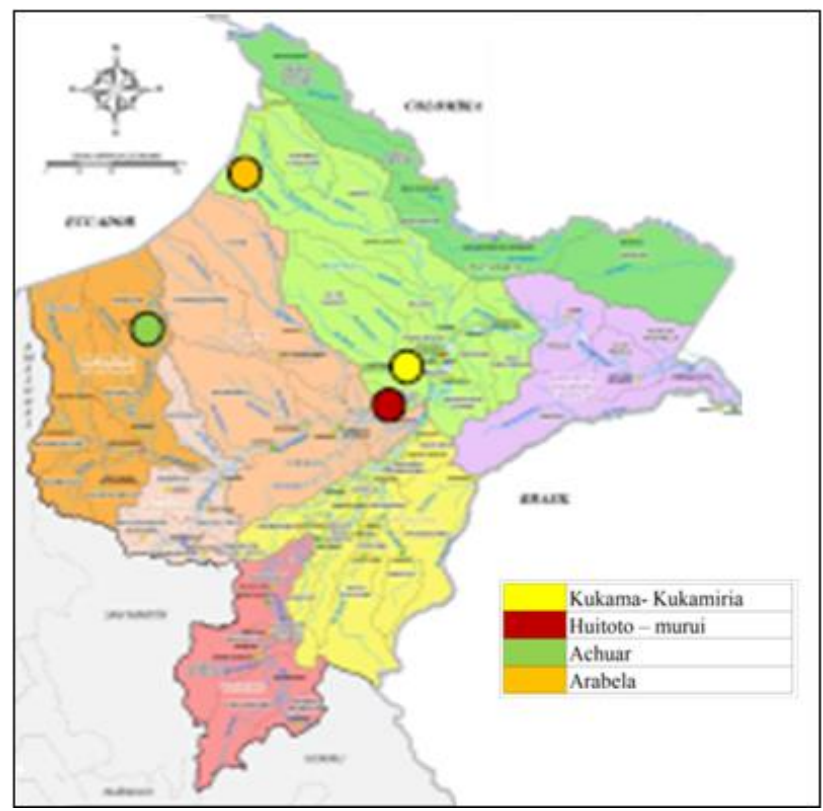

Figura 2. Mapa de localización de los Pueblos Indígenas participantes del estudio

En esta forma, a través de los instrumentos de recolección de datos se logró obtener información de interés para la investigación. Las cuatro comunidades intervenidas están asentadas a orillas de ríos. Todos son miembros en un pueblo indígena.
Los dos primeros pueblos indígenas (Kukama - Kukamiria y Huitoto-Murui) sostuvieron que no encuentran pecaríes en sus localidades y las dos últimas (Achuar y Arabela) alegan ver pecaríes cuando se van de cacería o en el camino a sus cultivos 
(chacras). Es importante precisar que las dos últimas comunidades están alejadas de la zona urbana (ver Figura 2). Ninguna de las cuatro comunidades ha comercializado pieles para almacenes o comerciantes intermediarios. Y las dos primeras sostienen que compran la carne de sajino de pequeños comerciantes una o dos veces al mes en el puerto de su localidad. Todos manifiestan no haber criado sajino, solo gallinas $\mathrm{y}$ algunos cerdos. Asimismo, manifiestan desconocimiento de la crianza de sajino. Es importante observar que solo el Pueblo Kukama- Kukamiria tienen títulos de propiedad otorgados por el Organismo de formalización de la propiedad informal COFOPRI en la comunidad de Nina Rumi, Rio Nanay. Los otros pueblos indígenas se mantienen en el sistema de tierras comunales.

\section{Diseño del área de crianza en las comunidades indígenas de estudio}

La primera actividad fue diseñar las instalaciones para la crianza (6) La mano de obra para la construcción fue aporte de cada familia criadora. Cada instalación utilizó los recursos naturales del bosque propiedad de cada pueblo indígena (madera en troza "sinchinas", hojas de "irapay" Lepidocaryum tenue, "bombonaje" Cardulovica palmata y "yarina" Phytelehas macrocarpa de acuerdo con la riqueza en cada bosque. Los recipientes para los alimentos y agua de los animales en el corral se han elaborado de madera pesada "batán" en dimensiones aproximadas de 0,20 x 0,30x 0,20 m. Además, en el interior del corral se han recreado parte de área del corral y zona de menor pendiente, una pequeña excavación de 0,60 x 0,50x0,20 m para la bañera de los animales.

Los animales (pecarí o sajinos criados en cautiverio) fueron transferidos del Centro Piloto de Zoocria de la Universidad Nacional de la Amazonia Peruana -UNAP. Los cajones que fueron diseñados para sajinos "jabas de transporte individual de animales" son de madera de 0,60x0,40x0,40 y llegan a la comunidad (1 macho: 2 hembras) para cada familia participante del estudio. En la Figura 3 se describe las jabas para transportarlos y Figura 4 se visualiza la forma en como transportarlos.

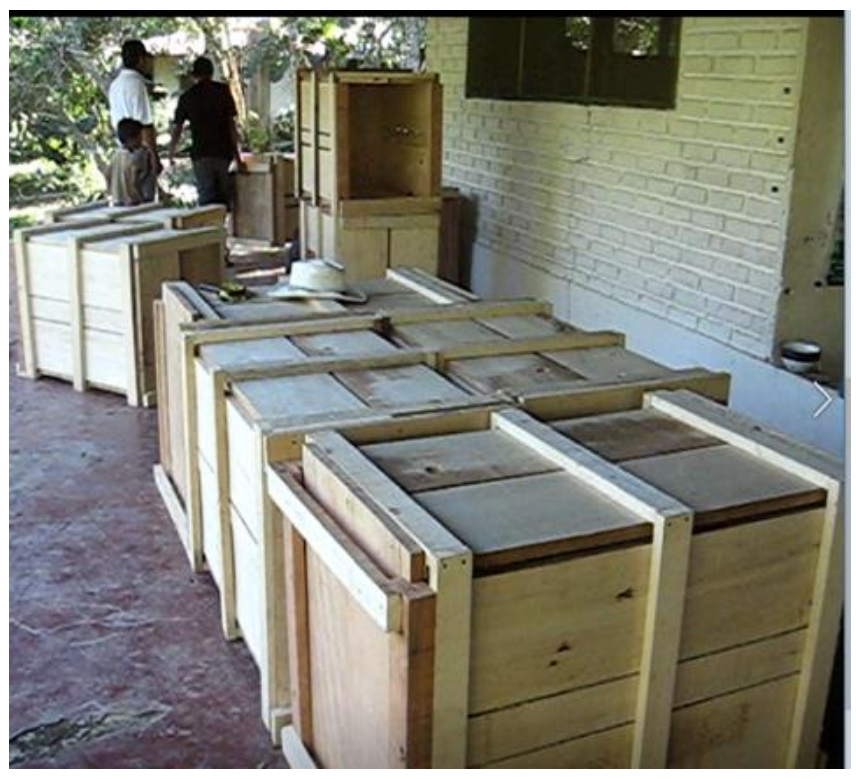

Figura 3. Jabas para el transporte de los pecaríes. 


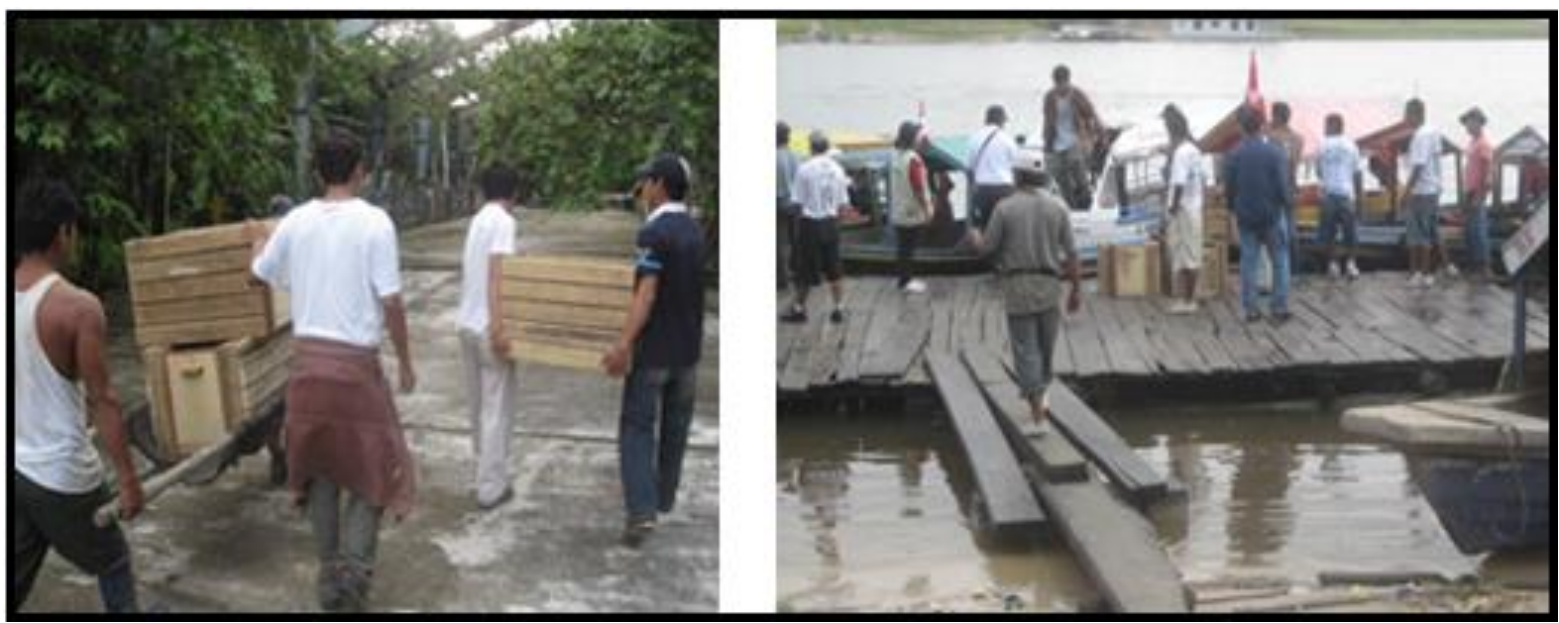

Figura 4. Forma de transportar los pecaríes desde el Centro Piloto hasta las zonas de estudio.

RESULTADOS pobladores han tomado posesión del lugar y

Al inicio de la actividad, la población reaccionó multitudinariamente $\mathrm{y}$ contribuyeron en el transporte de los animales, donde es la primera vez que ven pecaríes vivos, dóciles y que se van a criar en la comunidad. La población permaneció alrededor de los corrales. Durante toda la semana tienen visita las familias criadoras por motivo de la actividad y los animales. Los se observa incluso la presencia de pobladores de otros pueblos cercanos que llegan para observar a los animales y emerge la palabra sajinero. En el Figura 5, se muestra como los animales transferidos al término del proyecto (un año) se han reproducido por medio del primer parto en todas las comunidades, produciendo un incremento en la población.

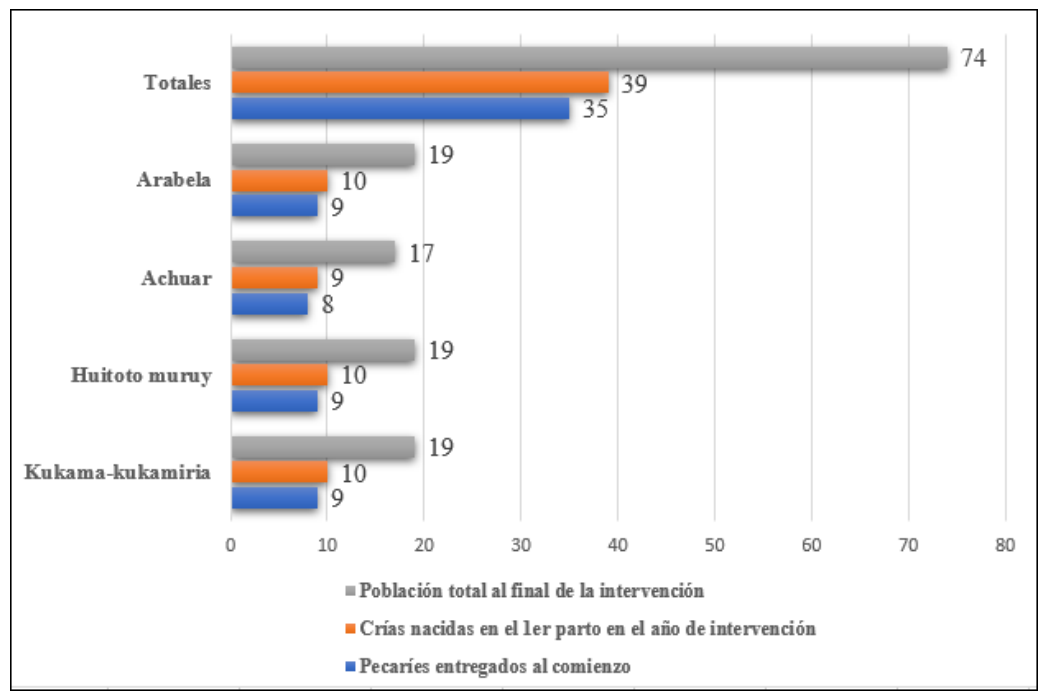

Figura 5. Población de animales transferidos al inicio y final de la intervención con logros de reproducción en cada pueblo indígena. 
Así mismo, en el Figura 6 se muestra la tendencia en lo esperado y lo obtenido en cada familia criadora de sajino participante en el estudio (logros en la crianza). Para ello, se ha creído conveniente utilizar los valores zootécnicos estimados en 126 partos en el Centro Piloto de Zoocria para la Amazonia- Iquitos, Perú (CPCM-UNAP) (13), como valores esperados. Demostrándose que los criadores en los pueblos indígenas han tenido éxito en la reproducción durante el estudio. En la Figura 7 se puede visualizar la población de pecaríes criados por las familias indígenas

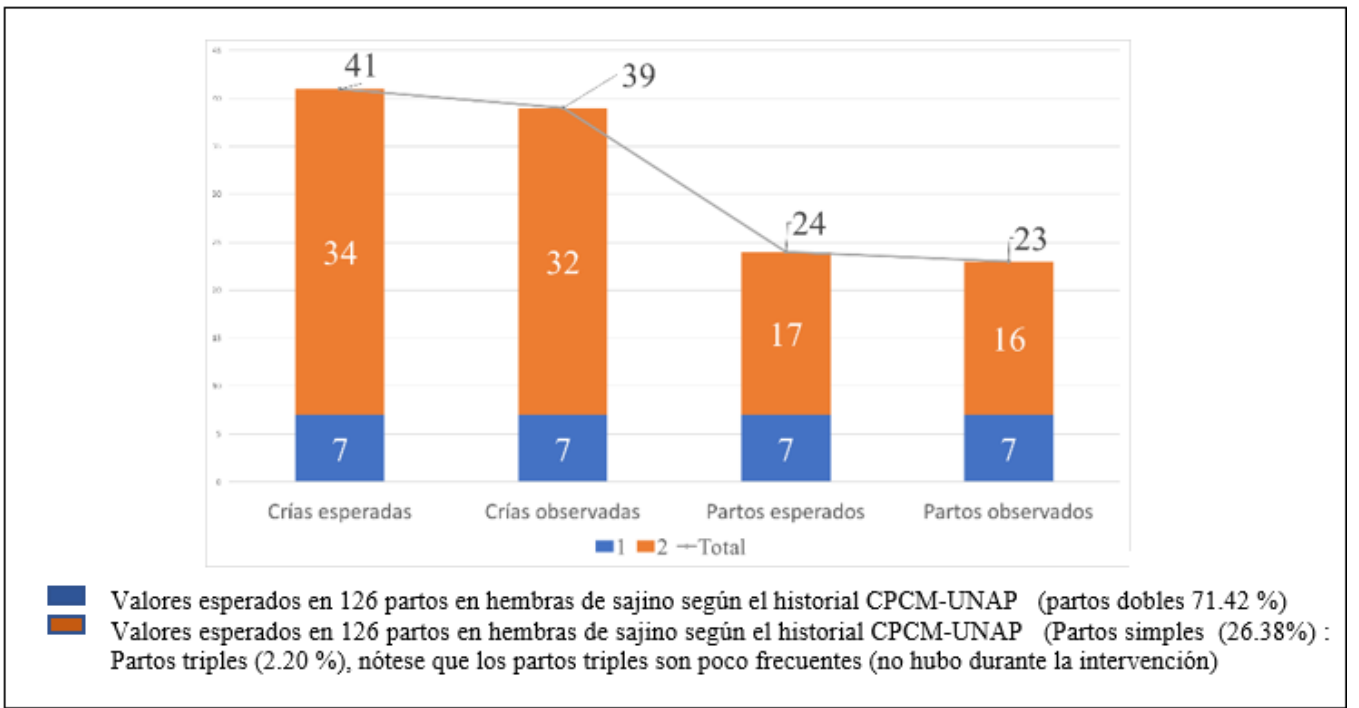

Figura 6. Producción de crías esperadas/ parto (estimados CPCM-UNAP) y Producción de crías observadas por criador en cada pueblo indígena

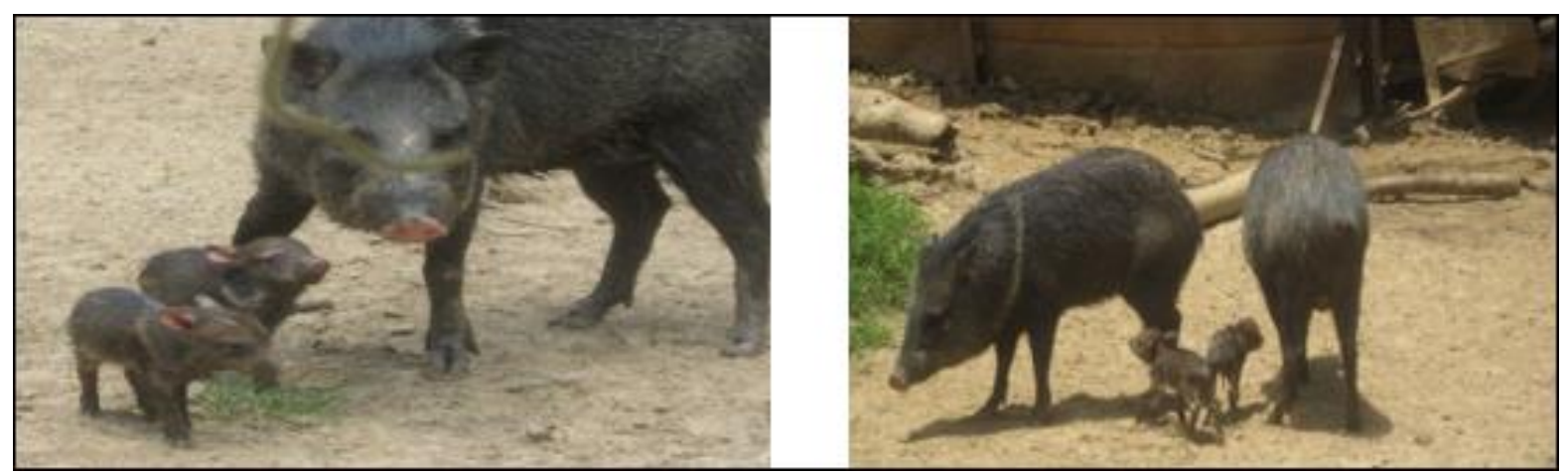

Figura 7. Población de pecaríes criados por las familias indígenas

Por otro lado, la alimentación de los animales en cada pueblo indígena se realizó según la diversidad de recursos alimenticios, del ecosistema de cada grupo étnico, para la alimentación de los pecaríes en cautiverio. En la tabla se muestra los nombres científicos de las plantas proporcionadas, tal como se muestra en la Tabla 1 y Figura 8. De ello, se puede determinar preferencia a frutos de palmeras, diversidad de recursos por Pueblo Indígena, destacando el pueblo Achuar y el conocimiento de frutos del bosque por su afinidad de ser cazadores de animales silvestres.

Alfa, Revista de Investigación en Ciencias Agronómicas y Veterinarias Vol. 4, NNro. 12, Septiembre - diciembre 2020 
Tabla 1. Insumos alimenticios administrados en crianza de pecarí en cautiverio en cuatro pueblos indígenas Achual, Kukama, Arabela y Huitoto muruy, respectivamente.

\begin{tabular}{|c|c|c|c|c|c|c|c|}
\hline \multicolumn{3}{|c|}{ Insumos Alimenticios administrados a pecarí } & \multirow[t]{2}{*}{ Uso } & \multicolumn{4}{|c|}{$\begin{array}{c}\text { Pueblo } \\
\text { Indígena } \\
\text { usuario }\end{array}$} \\
\hline Familia & Nombre científico & N. común & & 1 & 2 & 3 & 4 \\
\hline MYRTACEAE & Campomanesia lineatifolia $R Y P$ & Guayabilla & fruto & $x$ & & & \\
\hline EUPHORBIACEAE & Caryodendron orinocense $\mathrm{H}$. Karst. & Metahuayo & fruto & $x$ & & & \\
\hline EUPHORBIACEAE & Hevea guianensis Aubl. & shiringa & fruto & $x$ & & & \\
\hline ARECACEAE & Astrocaryum macrocalyx Burret & Huicungo & fruto & $x$ & & & \\
\hline ARECACEAE & Mauritia flexuosa L. f. & aguaje & fruto & $x$ & $\mathrm{x}$ & $x$ & $x$ \\
\hline ARECACEAE & Astrocaryum chambira Burret & chambira & fruto & $x$ & & & \\
\hline ARECACEAE & Bactris gasipaes Burret & pijuayo & fruto & $x$ & $x$ & $x$ & $x$ \\
\hline EUPHORBIACEAE & Manihot esculenta & yuca & tubérculo & $x$ & $x$ & $x$ & $x$ \\
\hline ARECACEAE & Elaeis oleífera & pumayarina & fruto & $x$ & & & \\
\hline FABACEAE & Inga $s p$ & shimbillo & fruto & & & $x$ & $x$ \\
\hline FABACEAE & Inga cinnamomea Sp ex Benth. & Shimbillo & fruto & $x$ & & $x$ & \\
\hline MORACEAE & Ficus insípida Willdenow ss. 247nsípida & oje & fruto & $x$ & & & $x$ \\
\hline EUPHORBIACEAE & Plukenetia volubilis $L$. & sachainchi & fruto & $x$ & & & \\
\hline CONVOLVULACEAE & Ipomoea batatas & camote & fruto & $x$ & & & \\
\hline POACEAE & Sacharum officinarum & Caña de azúcar & tallo & $x$ & $x$ & $x$ & $x$ \\
\hline ARECACEAE & Wettinia augusta Poepp. \& Endl. & Ponilla & fruto & $x$ & & & \\
\hline ARALIACEAE & Senefeldera macrophylla Ducke & Huangana Caspi & fruto & $x$ & & & \\
\hline ARECACEAE & Oenocarpus bataua sub sp. Bataua C. Martius & ungurahui & fruto & $x$ & $x$ & $x$ & $x$ \\
\hline ARECACEAE & Euterpe precatoria Martius & huasai & fruto & $x$ & & & $x$ \\
\hline LECYTHIDACEAE & Grias neuberthii Mcbride & sachamangua & fruto & $x$ & & & \\
\hline APOCYNACEAE & Parahancornia peruviana Monachino & Naranja podrida & fruta & $x$ & & & \\
\hline MORACEAE & Artocarpus altilis & Pan de árbol & fruto & $x$ & & & \\
\hline FABACEAE & Hymenaea courbaril $L$. & Azúcar huayo & fruto & $x$ & & & \\
\hline $\begin{array}{l}\text { Reyno animalia } \\
\text { AMPULLARIIDAE }\end{array}$ & Pomacea maculata & churo & Individuos & $x$ & & & \\
\hline MUSACEAE & Musa sp & Plátano & $\begin{array}{l}\text { Fruto y } \\
\text { tallo }\end{array}$ & & $x$ & $x$ & $x$ \\
\hline FABACEAE & Pueraria phaseoloides & kutzu & $\begin{array}{l}\text { Hojas y } \\
\text { tallo }\end{array}$ & & $x$ & & \\
\hline POACEAE & Zea mays & Maíz & $\begin{array}{l}\text { Mazorca } \\
\text { fresca } \\
\text { con } \\
\text { granos y } \\
\text { sin ellos }\end{array}$ & & $x$ & & $x$ \\
\hline
\end{tabular}




\begin{tabular}{|c|c|c|c|c|c|c|c|}
\hline \multicolumn{3}{|c|}{ Insumos Alimenticios administrados a pecarí } & \multirow[t]{2}{*}{ Uso } & \multicolumn{4}{|c|}{$\begin{array}{c}\text { Pueblo } \\
\text { Indígena } \\
\text { usuario }\end{array}$} \\
\hline Familia & Nombre científico & N. común & & 1 & 2 & 3 & 4 \\
\hline POACEAE & Zea mays multicolor & maíz multicolor & $\begin{array}{l}\text { Mazorca } \\
\text { fresca } \\
\text { con } \\
\text { granos y } \\
\text { sin ellos }\end{array}$ & & & $\mathrm{x}$ & \\
\hline CUCURBITACEAE & Cucurbita máxima & zapallo & fruto & & $x$ & $x$ & $\mathrm{x}$ \\
\hline FABACEAE & Eritrina $s p$ & amasisa & hojas & & & & $\mathrm{x}$ \\
\hline FABACEAE & Inga sp & cuaba & fruto & & & & $\mathrm{x}$ \\
\hline ICACINACEAE & Paraqueiba sericeae & umari & fruto & & & $x$ & $\mathrm{x}$ \\
\hline MALVACEAE & Theobroma grandiflorum & copoazú & fruto & & & & \\
\hline ARECACEAE & Elaeis guineensis & palma aceitera & fruto & & & & \\
\hline MIRTACEAE & Syzygium malaccenzis & poma rosa & fruto & & & & $\mathrm{x}$ \\
\hline ARECACEAE & Phytelephas macrocarpa Ruiz et Pav. & yarina & Fruto & & & $x$ & \\
\hline LECYTHIDACEAE & Eschweilera coriácea (DC.) S.A.Mori & machimango & fruto & & & $x$ & \\
\hline CARYOCARACEAE & Caryocar glabrumm (Aubl.) Persson & almendro & fruto & & & $x$ & $\mathrm{x}$ \\
\hline SAPOTACEAE & Pouteria macrophylla & quinilla & Fruto & & & & $\mathrm{x}$ \\
\hline OXALIDACEAE & Averrhoa carambola & carambola & fruto & & & & $\mathrm{x}$ \\
\hline MIRTACEAE & Psidium guajava & guayaba & fruto & & & & $\mathrm{x}$ \\
\hline ANACRADIACEAE & Spondias cutherea & taperibá & fruto & & & & $\mathrm{x}$ \\
\hline RUTACEAE & Citrus sinensis & naranja & fruto & & & & $\mathrm{x}$ \\
\hline LAURACEAE & Persea americana & palta & fruto & & & & $\mathrm{x}$ \\
\hline APOCYNACEAE & Couma macrocarpa & azúcar huayo & fruto & & & & $\mathrm{x}$ \\
\hline ARACEAE & Xanthosoma sp & huitina & bulbo & & & & $\mathrm{x}$ \\
\hline SAPOTACEAE & Pouteria caimito & caimito & fruto & & & & $\mathrm{x}$ \\
\hline MALVACEAE & Theobroma cacao & cacao & fruto & & & & $\mathrm{x}$ \\
\hline ANACARDIACEAE & Spondias mombin & ubos & fruto & & & & $x$ \\
\hline MALVACEAE & Matisia cordata & zapote & fruto & & & & $\mathrm{x}$ \\
\hline CUCURBITACEAE & Cucurbita melo & melón & fruto & & & & $\mathrm{x}$ \\
\hline CARICACEAE & Carica papaya & papaya & fruto & & & & $\mathrm{x}$ \\
\hline CHRYSOBALANACEAE & Supay ocote & Parinari & fruto & & & & $\mathrm{x}$ \\
\hline
\end{tabular}

Alfa, Revista de Investigación en Ciencias Agronómicas y Veterinarias Vol. 4, Nro. 12, Septiembre - diciembre 2020 


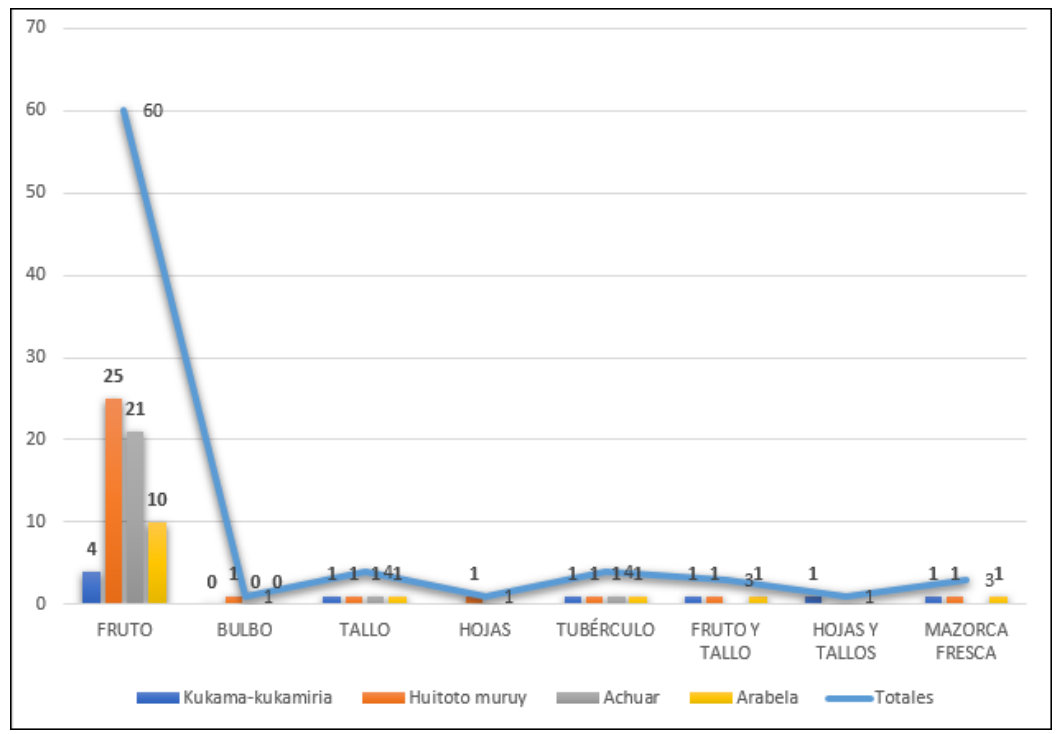

Figura 8. Diversidad de insumos alimenticios para sajino en la comunidad Achual. Kukama, Arabela y Huitoto-muruy, respectivamente.

DISCUSIÓN indígenas, se reportó al grupo de mamíferos

La vida de los pueblos indígenas en la Amazonía ha estado relacionada con la fauna silvestre desde hace siglos, siendo categorizada como de subsistencia, comercio y dependencia en el recurso faunístico como fuente de alimento. No obstante, hasta la fecha, las políticas de conservación (la caza como obstáculo), la caza como identidad cultural, dieta alimenticia, economía, prácticas sociales simbólicas practicadas por las comunidades indígenas o el gobierno con la expansión agrícola; no han logrado conciliar el desarrollo humano y la sostenibilidad de los ecosistemas (14-21). Esta investigación da una respuesta a priori para que se concilien los intereses para la conservación, identidad cultural, dieta alimenticia, economía, prácticas sociales simbólicas y expansión Agrícola pecuaria a partir del Pecarí tajacu cuyo manejo alimenticio y sanitario es a través del usufructo del bosque en pie.

Según estudios referidos a la caza de subsistencia en los pueblos indígenas y no como el más perseguido después continúan en la lista las aves y los reptiles. Asimismo, se establece una preferencia hacia animales grandes como primates y ungulados en el marco de teoría del forrajero óptimo. En este contexto, se permite ejercer presión sobre una fauna en particular (defaunación) que genera preocupaciones relacionadas a la seguridad alimentaria, la salud pública (epizootias), los derechos indígenas e incluso la seguridad nacional ante el tráfico de carne, pieles y animales vivos $(22,19,23,21,24,25)$. No obstante, esta investigación demuestra el estado de relación con la fauna silvestre en particular con el Pecarí tajacu y se corrobora que la caza como actividad le ha permitido conocer el bosque, las costumbres alimenticias de los pecaríes, sus lugares de refugio y el respeto a los animales. Esto ha sido de gran utilidad como saberes previos junto al conocimiento adquirido de forma oral por sus padres. Particularmente de los cazadores o agricultores, cuya participación ha sido fundamental durante la jornada de 
sensibilización, capacitación y sobre el manejo de esta especie en cautiverio en estos pueblos durante la intervención.

En estudios realizados en dos pueblos Baka en el Sureste de Camerún se muestra una situación similar a la de la Amazonia Peruana. Donde los pobladores sostienen una dicotomía, como: La interpretación de la caza, cambios ambientales y sus efectos sobre la estructura social donde subyacen principios de la conducta, respeto, identidad, regulaciones, tabúes y el bienestar al consumir la fauna, comercializar carne en sus poblados, pieles etc. (21). Y por otro lado, las medidas de conservación, los incentivos económicos del mercado y subproductos de la carne de animales silvestres de manera ilegal (26). Por lo que, recomienda que las políticas actuales deben reportar un entendimiento crítico y completo de las cosmovisiones y las reacciones a los cambios de las poblaciones locales en estos pueblos, en el marco del desarrollo sostenible (23). Así como, el caso de los Shuar de Ecuador, donde el animal de caza es considerado una especie de "pariente", una relación inestable y difícil que exige respeto mutuo (24). Los Makuna de Colombia, donde el cazador trata la caza como un conjunto potencial, que, debido a sus principales atributos -la mortalidad como la vida social y ceremonial, la intencionalidad, el conocimiento- no permite atribuirles identidades estables (27). En este particular, se parte de esta contextualización, por ello, se genera y valida una tecnología utilizando el recurso fauna silvestre (Pecari tajacu), alimentación de los animales utilizando el bosque y los saberes previos adquiridos e innatos de cada poblador, generando la adopción de animales criados en cautiverio para que los criadores (pobladores indígenas) se integren en otra forma de obtención del recurso (seguridad alimentaria todas las épocas del año).

Del mismo modo, la cacería en la amazonia es una actividad realizada exclusivamente por los hombres; y generalmente es realizada en solitario (28). Sin embargo, pueden darse ocasiones donde se practica de manera colectiva y muy ocasionalmente, con participación de la mujer $(26,24)$. Siendo así que, en muchas culturas, ser un cazador es esencial para obtener respeto, alcanzar la virilidad o empezar a establecerse con una familia. En los Ese Eja del sureste del Perú, se señala que la cacería está estrechamente relacionada con la identidad masculina y el prestigio. Está fuertemente sujeta a la imagen del cazador a través de la vida de la persona y está asociada con el prestigio que otorga el esfuerzo de la cacería ya que, por ejemplo, matar a un mamífero grande, como una sachavaca (Tapirus terrestris), garantiza carne para muchos en la comunidad (29-31). En el estudio realizado de cría de pecarí, se reporta como acontecimiento grato $\mathrm{y}$ fácil de aprender. La novedad de ver los animales del bosque criados en cautiverio (los niños nunca han visto un sajino vivo), las mujeres de la familia criadora participando de la actividad de alimentación y el valor o estatus social del criador al denotarlo, ya no como cazador, sino como sajinero (criador). Otro aspecto interesante es el comportamiento de la comunidad y de comunidades vecinas y la permanencia de estas en la proximidad al criadero ha sido un acto suigéneris $\mathrm{y}$ conmovedor.

En una compilación sobre manejo de fauna en pueblos indígenas se manifiesta que existe la necesidad de un seguimiento a las actividades de cacería para discutir el paradigma de enfoques científico-biológicos 
en el manejo de fauna silvestre que incluyan las dimensiones culturales y sociales para el aprovechamiento de fauna por estas comunidades locales $(32,31,33,27)$. En esta investigación se ha utilizado el método de IAP en donde el sujeto en estudio participa en la investigación, aporta conocimientos de cazador (sensibilización y posteriormente la capacitación en el manejo en cautiverio de la especie).El conocimiento previo de los pobladores indígenas (cazadores, agricultores o familia de cazadores) ahora criadores es de vital importancia dentro del proceso.

El uso de la estacionalidad fenológica de los frutos en el bosque (época de lluvias mayor abundancia $\mathrm{y}$ presente en su calendario de caza) le brinda un contexto de abundancia y disponibilidad de frutos y lo relaciona con el patrón de conducta de los animales y criadores hacen uso de este conocimiento para la crianza en cautiverio del pecarí. Una versión semejante lo describen en los Machiguenga del Parque Nacional Manu, quienes reportan una mayor actividad de cacería para primates durante la época de lluvias, cuando muchos frutos del bosque se diseminan y estos animales engordan (Ateles sp., Lagothrix sp.), ya que para ellos es costumbre cazarlos en esta época y prefieren no cazar en la sequía o vaciante (animales son pequeños y sin mucha grasa). De la misma manera, los Ese Eje de la Comunidad Nativa Sonene en el río Heath, manifiestan mayor caza de maquisapas (Ateles sp.) cuando estos están "kia sei" o con grasa acumulada, luego de los meses de lluvia (34-36, 33).

El manejo de fauna como "... el arte de usar la tierra para producir cosechas sostenidas anuales de animales silvestres" (37) las Prácticas simbólicas, que se hacen a partir de las restricciones o prohibiciones de cacería asociadas a criterios simbólicos de carácter conservacionista $(27,38)$. Este nos lleva, al manejo indirecto "ex situ" cuando un grupo realiza prácticas productivas que permiten la seguridad alimenticia y tienden a disminuir la presión sobre las poblaciones de animales silvestres y genera las prácticas tradicionales que mejoran la calidad o cantidad de un recurso; y las de cambio cultural, que requieren que se modifique la relación ser humano-naturaleza tanto a nivel simbólico como cotidiano para tener acceso a un recurso (Ejemplo crianza de vacunos en la amazonia) $(33,39)$. En este enfoque se asume que la crianza de pecaríes por los pueblos indígenas establece un paradigma de cazador a criador y demuestra que existen Actitudes Habilidades - Destrezas significativamente útiles para la crianza en cautiverio del pecarí por las familias criadoras miembros del pueblo indígena.

Es importante reconocer que la domesticación de animales y plantas generó cambios sustanciales en el comportamiento de las sociedades sedentarias. La nueva economía y la innovación tecnológica traen transformaciones sociales y políticas $(31,33)$. Nuestro estudio objetivamente validó la inserción y el cambio de percepción al lograr que los pobladores de pueblos indígenas logren criar Pecarí tajacu en cautiverio, es importante recordar que estos animales transferidos a las comunidades son planteles nacidos en cautividad (13) y que eran manejados con fines de domesticación. Asimismo, para el establecimiento de los módulos se capacito a los potenciales criadores en las diferentes áreas de manejo.

En este sentido, según Taylor y Bogdan (12) comentan que cuando establecen la metodología desde el trabajo comunitario integrado, basadas en el IAP, donde la expresión oral o escrita y la conducta observable se apoyan en principios $\mathrm{y}$ supuestos afines, presentes en todas las 
comunidades intervenidas. Siendo los sujetos actores sociales, poseedores de grandes capacidades para generar los propios cambios (31). Para este caso, las etapas propuestas con la metodología resultaron operacionalmente eficientes para el logro de los objetivos propuestos.

En el Perú es importante visibilizar la propiedad comunal y adaptarla a una tipología de unidad familiar de subsistencia (UFs), diferenciando estructuralmente el corte social, demográfico y económico e incluso geográfico (2). Coincidimos con esta afirmación y se debe incluir el aspecto legal en temas de crianzas de animales silvestres a nivel comunal. En este estudio se valida la crianza de pecarí por los pobladores. Sin embargo, queda por visibilizar las unidades familiares de subsistencia en base a animales silvestres. Debiendo ser reconocidos en el mismo nivel de las crianzas de animales domésticos foráneos (ganado vacuno, caprino, aves, conejo, cuyes, etc). A fin de apoyar las iniciativas con programas agropecuarios del gobierno de Perú, con subsidios o incentivos para estos nuevos ganaderos que no realizan ni Roza -TalaQuema, se aprovecha la pendiente del suelo y el alimento de los animales procede del "bosque en pie" por temporalidad estacionaria. Solo se requiere que el criador tenga la crianza en el traspatio o huerta para intensificar la interacción en la domesticación.

\section{CONCLUSIONES}

La caza de subsistencia para el poblador amazónico es importante social - cultural, pero está interesado en buscar formas de manejar sus recursos y conocer la naturaleza de su entorno.

Los pobladores de los pueblos indígenas participantes de la crianza de pecarí demostraron conocimientos previos útiles en la crianza del Pecarí tajacu. El interés en la crianza de pecarí en cautiverio demostrado y la existencia de paradigmas o cosmovisión acerca de este interés es conciliable, tomando en cuenta que demostraron habilidades y destrezas para la actividad y se estableció una jerarquía social "sajinero".

Un cazador es un sujeto altamente preparado para colectar frutas, hojas, tallos para el alimento del pecarí en cautiverio en la modalidad de crianzas familiares. Conoce la temporada de fructificación de los árboles.

La crianza de pecarí es una propuesta sostenible. No se Tala-Roza -Quema, es una producción limpia basada en el contexto de sostenibilidad del bosque e identidad cultural en cada pueblo.

Los tiempos para instalar una crianza, debe tener un tiempo de gracia de tres años como mínimo, a fin coadyuvar en el empoderamiento de la propuesta.

Debe habilitarse socialmente el concepto de Unidad Familiar de Subsistencia (UFs). Adaptando el sistema al concepto de propiedad comunal de los pueblos indígenas. Permitiendo ser sujetos y participes del desarrollo sostenible y ser beneficiarios del apoyo agrario del estado peruano.

\section{REFERENCIAS BIBLIOGRÁFICAS}

1. Penagos AG, \& Sabourin MË. Desigualdades y ruralidades en América Latina y el Caribe. Colombia: Centro Latinoamericano para el Desarrollo Rural - RIMISP. (2019)

2. Pintado Linares $\mathrm{M}$, \& Alvarado, QJ. Aportes de la agricultura familiar al sector agropecuario y una propuesta de tipología para su caracterización en el Perú. Lima: Trabajo de grado para optar el Título de Economista de la Pontificia Universidad Católica del Perú, Facultad de Economía. (2020) 
3. FAO. Panorama de la pobreza rural en América Latina y el Caribe 2018. Santiago de Chile: FAO. (2018)

4. INEI. IV Censo Nacional Agropecuario 2012. Censo. Lima: Instituto Nacional de Estadística e Informática. (2013)

5. Convenio sobre la diversidad biológica. Alternativas de medios de vida para el uso no sostenible de la carne de animales silvestres. Lima: Secretaria de Diversidad Biológica Perú. (2011)

6. Rengifo Pinedo E, \& Navarro Torres D. Crianza familiar del sajino o pecarí de collar en la Amazonia CETA. Iquitos, Perú: Editor CETA. (2002)

7. Brack EA. Perú diez mil años de domesticación. Lima, Perú: Editor Bruño. (2003)

8. Fang M., Tula G, Bodmer R, Puertas $P$, Pedro, P, Pedro M, Hayman D. Certificación de piel de pecaríes en la amazonia peruana. Una estrategia para la conservación y manejo de la fauna silvestre en la Amazonia peruana. (2008)

9. Ministerio del Ambiente del Perú. Dictamen de extracción no perjudicial DENV de los cueros de Pecarí tajacu y Tayassu pecarí en el Perú. 2017. Lima: Ministerio del Ambiente -MINAM, Dirección General de Biodiversidad Biológica. (2017)

10. Freitas Córdova JD. El comercio internacional de fauna silvestre en Loreto, Perú. Lima, Perú: Trabajo de grado presentado para optar al título de Magister en Conservación de Recursos Forestales de la Universidad Nacional Agraria. Obtenido de

http://repositorio.lamolina.edu.pe/bitstrea $\mathrm{m} /$ handle/UNALM/3861/freitas-cordovajoao-diego.pdf?sequence $=1$ \&isAllowed $=y$ (2019)

11. Rojas de Escalona B. Investigación cualitativa. Fundamentos y praxis. Caracas, Venezuela: FEDUPEL. Fondo Editorial de la Universidad Pedagógica Experimental Libertador. (2010).

12. Taylor S, \& Bogdan R. Introducción a los métodos cualitativos de Investigación. Paidós, Barcelona. (1990)
13. Rengifo Pinedo E, \& Flores Mere A. Estimación de los principales parámetros reproductivos y productivos del Pecarí tajacu, Linnaeus 1758 Pecarí de collar o sajino en cautiverio (Loreto, Perú). Lima, Perú: Trabajo de Postgrado. Universidad Nacional Agraria La Molina, Escuela de Postgrado - Especialidad Producción Animal. (2009)

14. Bennett E, \& Robinson G. Hunting for sustainability in tropical forest. New York: Columbia Universyty Press. (2000)

15. Bodmer R, Aquino $R, \&$ Puertas $P$. Alternativas de manejo para la Reserva Nacional Pacaya-Samiria: Un análisis sobre el uso sostenible de la caza. La Paz, Bolivia: Memoria. Congreso de Manejo fauna silvestre en la Amazonia. (1997)

16. Mayor P, Santos-Fita D, \& López M. Sostenibilidad en la Amazonia y cría de animales silvestres. Iquitos: Editor. CETA. (2007)

17. Nasi R, Brown D, Wilkie D, Bennett E, Tuntin C, Van Tol G, \& et al. Conservación y Utilización de recursos provenientes de la vida silvestre: La crisis de la carne de caza. Lima, Perú: Serie Técnica $N^{\circ} 33$. CDB CIFOR, UNALM. (2008)

18. Ojasti, \& Dallmeir F. Manejo de fauna Silvestre neotropical. Washington DC: Smithsonian Institution /MAB Biodiversity Program, SIMAB. (2000)

19. Redford K, \& Robinson JG. The game of choice: Patterns of indian and colonist hunting in the neotropics. American anthropologist, (1987); 89(3)

20. Ríos M, Dourojeanni M, \& Tovar A. La fauna y su aprovechamiento en Jenaro Herrera (Requena, Perú). Revista Forestal del Perú, (1974); 1(2)15-27

21. Romain D, Reyes-Garcia V, \& Bahuchet $S$. Ethnoecology of hunting in an empty forest: practices, local percepciones and social change among the Baka (Cameroo). Tesis. Barcelona: Universitat Autónoma de Barcelona, Institut de Ciencia i Tecnologia Ambientais

22. R K. (1992). The Empty Forest. Bioscience. (2017). 42., 412-422 
23. Rival L. Cerbatanas y lanzas. La significación social de las elecciones tecnológicas de los Huaroni. En P. Descola, \& G. Pálsson, Naturaleza y Sociedad. Siglo XXI E. (2001)

24. Torralba A. Los Sharanahua. Lima, Perú: Misiones dominica. (1986)

25. Vickers W. Hunting yields and game compositium over ten years in an Amazonian village. En J. Robinson, \& K. Redford, Neotropical wildlife use and conservation (1997); 977-982. University of Chicago Press. 4

26. Descola P. Las Cosmologías de los Indios de la Amazonia. Zainak; (1998). (17)219227

27. Ulloa A, Rubio H, \& Campos C. Conceptos y metodologías para la preselección y análisis de alternativas de manejo de fauna de caza con indígenas Embera en el Parque Nacional Utria Choco, Colombia. En C. A. Campos, H. Ulloa, \& Rubio, Manejo de fauna con comunidades rurales (1996); 19-48. Natura

28. Kensinger K. Los Huni Kuin. En F. Santos, \& F. Barclay, Guía etnográfica de la alta amazonía (1998); 2-124. Quito, Ecuador: Smithsonian tropical Research Institute. 3

29. Alexiades M. Ethnobotany of the Ese Eja: Plants, Health, and change in a Amazoniam. Society. New York University, (1999); 125-136

30. Morgado Garcia A. De la visión emblemática a la visión desencantada: Los animales en el mundo hispánico. Siglo XVII y XVIII. (2015)

31. Olazával HA. Los orígenes de la agricultura. Nuevos paradigmas. Arqueología Lima, Perú. (2014). 18(33), 53-86

32. Da silva M, Shepard G, \& Yu D. Conservations implications of primate hunting practices among the Matsigenka of manu National Park. Neotropical Primates. (2005); 13(2)31-36

33. Sanvicente, L. M., Vargas, L. S., Bustamante, G. A., \& Jaramillo, V. L. La crianza de cerdos en vida libre y pecaríes silvestres en zonas de transición de áreas protegidas del sureste de México. Archivos de zootecnia Journal website https://www.uco.es/ucopress/az/index. php/az/. (2020); 69(266)216-224

34. Moreno E, \& Ahumada M. Animales y humanos en las cumbres de Ancasti (Siglo VIII y IX DC) Paisajes campesinos y recursos locales. Archaeofauna; (2018); (27)195-208

35. Nava Hernández, G., Aldasoro Maya, E. M., Perezgrovas Garza, R., \& Vera cortés, G. Interacciones del ser humano con animales de traspatio: Un estudio desde la etnoveterinaria en Tabasco, México. Tesis de Postgrado en Ciencias en Recursos Naturales y Desarrollo RuralColegio Frontera Sur, Departamento de Agricultura, sociedad y ambiente. Sección Ciencias Naturales e Ingeniería. (2018)

36. Pérez Ojeda del Arco A, \& Vásquez Ruesta P. Cacería de subsistencia y etnoecología asociada en una comunidad nativa Amahuaca de la Provincia de PurúsUcayali, Perú. Lima: Tesis para optar el Título de Ingeniero Forestal de la Universidad Nacional Agraria La Molina, Facultad de Ingeniería Forestal. (s.f.)

37. Ojasti J. Utilización de la fauna silvestre en América Latina. Situación y perspectivas para un manejo sostenible. Roma, Italia: Guía FAO. Organización de las Naciones Unidas para la Alimentación y la Agricultura. Reporte No.: 25. (1993)

38. Ulloa A. ¿Ser Humano? ¿Ser Animal? Rostros culturales de la fauna: Relaciones entre los humanos y los animales en el contexto colombiano. Fundación Natura Colombia. (2002). (1)9-29

39. Vásquez Ruesta $P, \&$ Tovar $C$. La fauna en la reserva nacional Pacaya - Samiria: Una Guía para el manejo comunal. Centro de Datos para la conservación UNALM/ Pro Naturaleza/ The Nature Conservancy/ USAID; (2007); (120) 